\title{
REVIEW
}

\section{Brentuximab Vedotin in CD30+ Lymphomas}

Guilherme Fleury Perini · Barbara Pro

To view enhanced content go to www.biologicstherapy-open.com

Received: November 26, 2012 / Published online: March 1, 2013

(c) The Author(s) 2013. This article is published with open access at Springerlink.com

\section{ABSTRACT}

Monoclonal antibodies (mAb) have become an effective treatment strategy for hematologic malignancies. CD30 is a rational target for therapy due to its limited expression on normal tissues and the strong and uniform expression on malignant cells in classical Hodgkin's lymphoma (cHL) and anaplastic large-cell lymphoma (ALCL). Brentuximab vedotin, an anti-CD30 antibody-drug conjugate, utilizes the targeting properties of $\mathrm{mAb}$ to deliver a cytotoxic agent inside the malignant cell. Brentuximab vedotin has significant clinical activity in patients with relapsed or refractory cHL and

G. F. Perini

Hospital Israelita Albert Einstein, Sao Paulo, SP, Brazil

B. Pro $(\bowtie)$

Fox Chase Cancer Center, Lymphoma Service, Philadelphia, PA, USA

e-mail: Barbara.Pro@jefferson.edu

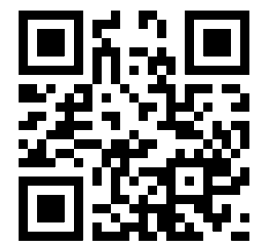

Enhanced content for this article is

available on the journal web site:

www.biologicstherapy-open.com relapsed or refractory ALCL, and has the potential to represent a significant advance in modern oncology.

Keywords: Anaplastic large-cell lymphoma; Antibody-drug conjugate; Brentuximab vedotin; CD30; Hodgkin's lymphoma; Monomethylauristatin E; Oncology

\section{INTRODUCTION}

CD30 is a prosurvival receptor, which belongs to the tumor necrosis factor receptor (TNFR) superfamily [1]. Although CD30 was recognized in the early 1980s as an important marker for Hodgkin Reed-Sternberg cells (HRS), it is now known that CD30 is also expressed in other B cell and $\mathrm{T}$ cell non-Hodgkin's lymphomas (NHL), as well as in a normal subset of activated B- and T-natural killer (NK) cells and activated monocytes [2].

Two lymphoid neoplasms are characterized by strong and uniform expression of CD30: Hodgkin's lymphomas (HL) and anaplastic large cell lymphoma (ALCL). HL accounts for approximately $11 \%$ of all malignant 
lymphomas, and is further classified in two major subtypes: classical (cHL) and nodular lymphocyte predominant HL [3]. In cHL, modern treatment combining chemotherapy and radiotherapy can cure the majority of patients. However, $10-30 \%$ of patients may progress or relapse, depending on baseline risk factors and type of treatment [4]. Up to 50\% of these patients can still be cured with high-dose chemotherapy and autologous stem cell transplantation (ASCT). The prognosis of patients who are primary refractory or relapse after ASCT is poor, with a median overall survival (OS) of approximately 2 years, constituting a highly unmet medical need [5].

Systemic ALCL (sALCL) is an aggressive T cell lymphoma that represents approximately 2-3\% of all lymphoid neoplasms. ALCL can be further classified on the basis of the expression of the anaplastic lymphoma kinase (ALK) protein. Patients with ALK-positive ALCL and no risk factors have responses similar or better than patients with B cell lymphomas when treated with conventional anthracycline-based chemotherapy. However, patients with ALKnegative ALCL tend to have low response rates and up to $60 \%$ of patients may relapse after front-line therapy [6]. High-dose therapy and ASCT may result in long-term remission in $30-40 \%$ of patients with chemosensitive disease [7].

\section{FIRST GENERATION ANTI-CD30 ANTIBODIES}

Rituximab, an anti-CD20 monoclonal antibody $(\mathrm{mAb})$, has consistently proved to increase the response rates and disease-free survival in almost all B cell neoplasms [8]. The success of an immunotherapy-based strategy in lymphomas led to intense research for other targets for mAbs, including CD30. A number of anti-CD30 mAbs have been developed, including SGN-30, MDX-060, and XmAb2513.

SGN-30 (also known as cAC10) is a chimeric anti-CD30 antibody constructed from the variable regions of the anti-CD30 murine monoclonal AC10 and the human gamma 1 heavy chain and kappa light chain constant regions. In a phase 1 study, 24 patients with CD30+ malignancies, including 21 patients with HL and 3 patients with CD30+ NHL, were treated with escalating doses of SGN-30. Treatment was well tolerated and no maximum tolerated dose (MTD) was reached. However, although well tolerated, responses were modest: one patient with cutaneous ALCL achieved a complete response (CR) and six patients, including four patients with $\mathrm{HL}$, achieved stable disease, with durations up to 16 months [9].

In an open-label, phase 2 study, 79 patients with recurrent or refractory HL $(n=38)$ or sALCL $(n=41)$ received weekly infusion of SGN-30 for 6 weeks, with the initial 40 patients receiving $6 \mathrm{mg} / \mathrm{kg}$ weekly, and the latter $12 \mathrm{mg} / \mathrm{kg}$ weekly [10]. Although proved safe, with only mild adverse events, objective responses were observed only in the ALCL group, in which two patients achieved a CR and five patients achieved a partial response $(\mathrm{PR})$, with response durations ranging from 27 to $1,460+$ days. No objective responses were observed in the HL group, and only 11 patients (29\%) achieved stable disease. The role of SGN30 in cutaneous ALCL was further tested in a phase 2 trial [11], showing an overall response rate (ORR) of 70\% (16 of 23 patients).

Since modest responses were observed with SGN-30 in HL, and based on the synergistic activity observed in preclinical models when SGN-30 was combined with cytotoxic agents active in HL, a phase 2 study explored the 
feasibility and efficacy of SGN-30 in combination with GVD (gemcitabine, vinorelbine, and pegylate liposomal doxorubicin). Initially, 16 patients were treated with SGN-30 plus GVD, and in a second portion of the study, patients were randomized to receive GVD with either SGN-30 or placebo. However, this trial was prematurely closed due to an increased pulmonary toxicity observed in patients treated with SGN-30 and GVD [12].

MDX-060 is a fully human anti-CD30 immunoglobulin $\mathrm{G} 1 \kappa \quad \mathrm{mAb}$ with greater antibody-dependent, cell-mediated cytotoxicity. In a sequential phase $1 / 2$ study, 72 patients with HL $(n=63)$, ALCL $(n=7)$, and CD30+ T cell lymphoma $(n=2)$ were enrolled. MDX-060 was well tolerated, with no MTD identified. However, limited single-agent activity was observed, with only 6 patients with clinical responses and 25 patients with stable disease [13]. The second generation anti-CD30 antibodies (MDX-1401 and XmAb2513) have modified Fc (fragment, crystallizable) regions to increase efficacy. Preliminary results from phase 2 studies show good tolerability but limited efficacy $[14,15]$.

\section{BRENTUXIMAB VEDOTIN}

Since minimal activity was observed in trials of first-generation "naked" anti-CD30 antibodies, and the combination of SGN-30 with cytotoxic agents resulted in significant toxicity, new strategies for immunotherapy against CD30 were tested including the development of an antibody-drug conjugate (ADC).

The combination of the chimeric anti-CD30 antibody cAC10 with monomethylauristatin E (MMAE), a potent antimitotic agent, led to the development of brentuximab vedotin [16]. A protease-cleavable linker was used to attach
MMAE to cAC10, offering significant stability, with less than $2 \%$ release of the drug from the $\mathrm{mAb}$ after 10-day incubation in human plasma [17]. Brentuximab vedotin has a well defined structure with an average of four drugs per $\mathrm{mAb}$ and no free MMAE in the drug product.

After targeting the CD30 receptor, brentuximab vedotin is internalized into the cells and the peptide linker is subsequently cleaved by lysosomal enzymes, releasing MMAE inside the cells. MMAE blocks the polymerization of tubulin, resulting in $\mathrm{G} 2 / \mathrm{M}$ phase growth arrest and apoptotic death. In vivo, brentuximab vedotin inhibits proliferation, induces apoptosis, and complete tumor regression in mouse xenograft models of both HL and ALCL, with improved efficacy relative to the unconjugated antibody [18].

In vivo studies in animals suggest that only a fraction of MMAE released from the ADC is metabolized, mainly via oxidation by CYP3A4/ 5. The elimination of MMAE occurs both in urine and feces, the latter being responsible for $72 \%$ of the total MMAE recovered. Interestingly, the elimination of MMAE appears to be limited by its rate of release from the ADC. There are currently no studies on dose adjustment for special populations, such as elderly patients, or patients with renal or hepatic impairment.

\section{CLINICAL STUDIES IN CD30+ MALIGNANCIES}

\section{Phase 1 Studies}

In the initial phase 1 dose-escalation study, 45 patients with confirmed relapsed/refractory CD30+ hematologic malignancies (42 patients with CHL, 2 patients with SALCL, and 1 patient with $\mathrm{CD} 30+$ angioimmunoblastic $\mathrm{T}$ cell lymphoma) were enrolled. The median age 
was 36 years and the median number of chemotherapy regimens was 3, with $73 \%$ of patients having failed a prior ASCT. Brentuximab vedotin was administered intravenously every 3 weeks at doses escalating from 0.1 to $3.6 \mathrm{mg} / \mathrm{kg}$, with no premedication needed. The MTD for dosing every 3 weeks was defined as $1.8 \mathrm{mg} / \mathrm{kg}$ and the dose-limiting toxicities were febrile neutropenia, prostatitis, and hyperglycemia. Other common adverse events included fatigue, peripheral neuropathy (PN), nausea, diarrhea, neutropenia, and pyrexia, but were usually grade 1 or 2 in severity [19].

Objective responses were observed in 17 patients, including 11 CRs. Tumor regression, assessed by computed tomography, was observed in 36 of 42 evaluable patients (86\%). Furthermore, $81 \%$ of patients with B symptoms had resolution of symptoms following treatment, regardless of response status. For patients receiving the MTD, the ORR was 50\%.

In another phase 1 study [20], 44 patients with relapsed/refractory $\mathrm{CD} 30+$ malignancies (38 patients with cHL, 5 patients with ALCL, and 1 patient with peripheral $\mathrm{T}$ cell lymphoma) were enrolled to receive a different schedule of brentuximab vedotin, with weekly doses of $0.4-1.4 \mathrm{mg} / \mathrm{kg}$ for 3 out of 4 weeks in each cycle. Significant clinical activity was observed with tumor regression observed in $85 \%$ of all patients, with a CR rate of $34 \%$. However, in comparison with the 3 week schedule, there was a significant increase in neuropathy, with $10 \%$ of the patients experiencing grade 3 neuropathy.

\section{Phase 2 Studies}

Due to encouraging results in the phase 1 trials, two separate phase 2 trials were conducted in patients with cHL and sALCL. In a pivotal, open-label, single-arm, phase 2 trial, patients with confirmed diagnosis of relapsed or refractory HL after ASCT received brentuximab vedotin at the dose of $1.8 \mathrm{mg} / \mathrm{kg}$ intravenously every 3 weeks for a maximum of 16 infusions [21]. A total of 102 patients were enrolled with a median age of 31 years (range, 15-77 years). Over $70 \%$ of patients had primary refractory disease and $42 \%$ had disease refractory to the most recent therapy, with a median number of prior therapies of 3.5 (range, 1-13) excluding ASCT. The ORR was $75 \%$, and $34 \%$ of all patients achieved a CR. Furthermore, tumor reductions were observed in nearly all patients (94\%). The median duration of response was 6.7 months, and was longer (20.5 months) for patients who achieved a CR. The estimated 12 -month overall survival was $89 \%$ and the estimated median progression-free survival (PFS) was 5.6 months, although patients achieving a CR had considerably longer (21.7 months) PFS. As observed in the phase 1 studies, the most common adverse events were peripheral sensory neuropathy (42\%), nausea (35\%), fatigue (34\%), neutropenia (19\%), diarrhea (18\%), pyrexia (14\%), vomiting (13\%), arthralgia (12\%), pruritus (12\%), myalgia (11\%), peripheral motor neuropathy (11\%), and alopecia (10\%). PN was the most common adverse effect leading to treatment discontinuation. However, complete resolution of all events of peripheral neuropathy was observed in $50 \%$ of the patients [21].

In another multicenter, open-label, phase 2 trial, 58 patients with relapsed or refractory ALCL after at least one prior therapy with curative intent [CHOP (cyclophosphamide, hydroxydaunorubicin, oncovin, prednisone) in most of patients], were enrolled [22]. Brentuximab vedotin was administered intravenously once every 3 weeks, for up to 16 doses. The median age was 52 years (range, $14-76$ years) and $72 \%$ of patients had 
ALK-negative disease, with most patients (62\%) being primary refractory to front-line treatment. The median number of prior regimens was 2 (range, 1-6), with 26\% patients failing ASCT before enrollment. Tumor reductions were observed in $97 \%$ of patients, with an ORR of $86 \%$, and $57 \%$ of patients achieving CR. The median duration of response was 12.6 months for all patients, and 13.2 months for patients in CR.

The responses were similar for patients with ALK-negative (ORR 88\%, CR 52\%) and ALK-positive (ORR 81\%, CR 69\%) disease. The median PFS time was 13.3 months for responding patients and 14.6 months for patients in CR. At the time of analysis, the median OS had not yet been reached, but the estimated 12-month survival rate was 70\%. Interestingly, this study analyzed subgroups of patients who had subsequent stem cell transplantation (SCT). For the 22 patients who achieved a CR and did not have a subsequent SCT, the median duration of response was 12.6 months, compared with 13.2 months for patients who subsequently had an allogeneic SCT and a median not reached for the five patients who had a subsequent autologous SCT in CR [22].

\section{SAFETY PROFILE OF BRENTUXIMAB VEDOTIN}

Generally, outpatient treatment with brentuximab vedotin is well tolerated. As with other chimeric mAbs, infusion-related reactions are observed, but are manageable. Premedication is usually necessary only for patients who experienced prior infusionrelated reactions. Other common side effects include nausea, fatigue, neutropenia, diarrhea, pyrexia, vomiting, arthralgia, pruritus, myalgia, and alopecia. Most adverse events are manageable through standard supportive care and are typically grade 1 or 2 .

$\mathrm{PN}$ is the most clinically significant adverse event associated with brentuximab vedotin treatment. PN is cumulative and dosedependent, consistent with that observed with other antimicrotubule agents. Most patients develop sensory neuropathy, characterized by numbness and tingling of fingers and toes, but peripheral motor neuropathy was observed in $9 \%$ of patients in one of the phase 1 trials. In the pivotal phase 2 study for HL, 56 patients experienced $\mathrm{PN}$, and these events were typically grade 1 and $2 ; 11 \%$ of patients had grade 3 peripheral neuropathy, and there were no grade 4 events [21]. Only nine of these patients had to discontinue treatment. Similarly, in the ALCL study, $41 \%$ of patients presented with $\mathrm{PN}$, with $12 \%$ of patients with grade 3 severity [22].

Complete resolution of symptoms occurred in $48 \%$ of patients and the median time to improvement or resolution was 9.9 weeks. For patients who develop grade 2 or 3 neuropathy, it is recommended that treatment be withheld until neuropathy improves to grade 1 , and the dose should then be decreased to $1.2 \mathrm{mg} / \mathrm{kg}$.

Since severe pulmonary toxicity was observed in the phase $1 / 2$ study of SGN-30 combined with gemcitabine, vinorelbine, and lyposomal doxorubicin, there was a concern that pulmonary toxicity could be observed with brentuximab vedotin. In a phase 1 study by Younes et al. [23], the combination of brentuximab vedotin with doxorubicin, bleomycin, vinblastine, dacarbazine (ABVD) as frontline therapy of patients with advanced-stage cHL resulted in pulmonary toxicity in 7 of 19 patients, leading to a formal warning by the US Food and Drug Administration (FDA) against the use of brentuximab vedotin with bleomycin. 
The exact mechanism of pulmonary toxicity with SGN-30 or brentuximab vedotin when used in combination with chemotherapy is not known. In the SGN-30 + GVD trial, five patients experienced grades 3-5 pulmonary toxicities, and were treated with broadspectrum antibiotics and concomitant highdose steroids. Although an infectious cause could be proved postmortem in one patient, there is a possible genetic cause, since distinct FC $\gamma$ RIIa and FC $\gamma$ RIIIa polymorphisms was observed in these patients [12].

Since the approval of brentuximab vedotin, two confirmed cases and one suspected case of progressive multifocal leukoencephalopathy (PML) were reported [24], and patients with new-onset central nervous system abnormalities should be evaluated promptly. Due to the serious nature of PML a new "Boxed Warning" highlighting this potential risk was added to the drug label [25]. PML has been observed with the use of other mAbs, especially with rituximab. Although with the latter, a possible $\mathrm{B}$ cell depletion and consequent immunosuppression can play a causative role, it is known that activated B- and $\mathrm{T}$ cells express CD30, and an anti-CD30 therapy can possibly lead to dysregulation of the immune system.

\section{CURRENT STUDIES AND FUTURE DIRECTIONS}

Based on the encouraging results from phase 2 studies, brentuximab vedotin is currently being investigated in different disease settings. Given the high response rate in the relapsed setting and the manageable toxicity profile, incorporating brentuximab vedotin into firstline therapy represents an attractive option. Ansell et al. [23] recently presented the interim results of a phase 1 study of brentuximab vedotin combined with ABVD or AVD (doxorubicin, vinblastine, dacarbazine) in patients with newly diagnosed advanced-stage cHL. In total, 47 patients were evaluated after treatment, and only 7 patients had to discontinue therapy due to adverse events. Bleomycin was discontinued in 11 patients due to pulmonary toxicity, and all other adverse events were mild and manageable. The objective response was 96\%, with 21 of 22 patients in the ABVD and 23 of 25 patients in the AVD group achieving a CR.

Multiple studies are being conducted with brentuximab in different scenarios. A phase 3 trial of brentuximab vedotin combined with $A V D$ versus $A B V D$ is being planned. In addition, a phase 3 placebo-controlled trial is evaluating the efficacy of brentuximab vedotin given as maintenance therapy in high-risk patients after ASCT. An estimated 322 patients completed accrual and will receive best supportive care following ASCT plus either brentuximab vedotin $1.8 \mathrm{mg} / \mathrm{kg}$ every 3 weeks, or placebo. Gopal et al. [26] recently showed that brentuximab vedotin is safe in patients relapsing after allogeneic SCT, with clinical responses in $50 \%$ of patients, and modest adverse events.

Brentuximab vedotin is also being tested in other CD30+ nonlymphomatous malignancies [27], T/NK lymphomas [28] and in steroidrefractory graft versus host disease [29].

\section{DISCUSSION}

The introduction of a safe and effective antiCD30 ADC has written another chapter in one of the most successful histories in modern oncology. HL, previously a lethal disease, is now highly curable when treated with multiagent chemotherapy regimens, with or without 
radiation therapy. For those patients not responding to first-line therapy, high-dose chemotherapy followed by ASCT can cure almost 50\% of patients [5]. However, a fraction of patients will not benefit from this strategy, in particular patients with primary refractory or chemoresistant disease, and those not eligible for ASCT.

The challenge in making a successful history more successful has led to intense research in cHL, especially in the relapse/refractory setting. Moreover, the fact that conventional chemotherapy may fail to eradicate the disease, and that the interaction between HRS and the surrounding stroma may play a major role, have brought new targets to attention. Histone deacetylase inhibitors [30], mammalian target of rapamycin inhibitors [31], and immunomodulatory agents [32] have shown promising results. Furthermore, rituximab has shown activity in cHL, regardless of CD20 expression by HRS, possibly by targeting the reactive B-cells which support the HRS cells [33].

CD30 represents the most attractive target in cHL, given the uniform and strong expression on tumor cells and the limited expression in normal tissues. However, early phase 1-2 clinical trials with "naked" anti-CD30 were disappointing, with almost no objective clinical responses.

The idea of using an ADC to selectively deliver cytotoxic drugs to the target malignant cells, like a "Trojan horse," is very interesting, and has been proved possible with the anti-CD33 ADC gemtuzumab ozogamicin. Studies with the novel ADC brentuximab vedotin demonstrated impressive clinical efficacy in heavily pretreated patients. These results are even more impressive if we consider that these patients have failed an ASCT. Moreover, these results represent proof of principle that the ADC methodology can overcome some factors that have limited the efficacy of conventional immunotherapy in cHL. Furthermore, the response rates observed with brentuximab vedotin are superior to other novel single agents [30-32].

The prognosis of patients with sALCL treated with conventional therapy is relatively poor, especially if the disease is ALK-negative. For these patients, the benefit of ASCT as salvage therapy is even more subtle, and patients not achieving a CR or at least a good PR before ASCT usually have poor prognosis. Moreover, patients failing ASCT have few options. New drugs have received accelerated approvals from the FDA for treatment of relapsed/refractory peripheral $\mathrm{T}$ cell lymphoma, including ALCL. However, in phase 2 trials that preceded the approval of these drugs (pralatrexate and romidepsin), the ORR rates were less than $30 \%$, with less than $15 \%$ of patients achieving a CR [34, 35].

Thus, the options for ALCL patients failing first-line treatment are even scarcer, with inferior outcomes. Therefore, brentuximab vedotin has proven the best option for those patients, even before ASCT, especially when less than a CR is achieved by salvage therapy. For those patients, brentuximab vedotin is a rational bridge before transplant.

Finally, the next step when treating cHL and ALCL patients in CR after treatment with brentuximab vedotin is unclear. Although allogeneic transplant is possible, retreatment may also be possible. In a case series, patients with $\mathrm{CD} 30+$ malignancies underwent retreatment, and objective responses were observed in six of eight patients [36].

\section{CONCLUSION}

Brentuximab vedotin is an anti-CD30 ADC with proven efficacy in patients with relapsed or refractory HL and relapsed or refractory sALCL. 
Other $\mathrm{CD} 30+$ malignancies have also shown response in case reports/series. Due to a favorable toxicity profile, brentuximab vedotin is now an excellent option for HL failing an ASCT and for ALCL patients after one prior course of multi-agent chemotherapy regimen.

\section{ACKNOWLEDGMENTS}

Seattle Genetics were offered the opportunity to review the paper for scientific accuracy prior to peer review. Dr. Pro is the guarantor for this article, and takes responsibility for the integrity of the work as a whole.

Conflict of interest. Guilherme Fleury Perini declares no conflict of interest. Barbara Pro has received a research grant from Seattle Genetics.

Open Access. This article is distributed under the terms of the Creative Commons Attribution Noncommercial License which permits any noncommercial use, distribution, and reproduction in any medium, provided the original author(s) and the source are credited.

\section{REFERENCES}

1. Clodi K, Younes A. Reed-Sternberg cells and the TNF family of receptors/ligands. Leuk Lymphoma. 1997;27:195-205.

2. Younes A, Consoli U, Snell V, et al. CD30 ligand in lymphoma patients with CD30+ tumors. J Clin Oncol. 1997; 15:3355-62.

3. Swerdlow SH, Campo E, Harris NL, et al. WHO classification of tumours of haematopoietic and lymphoid tissues. Lyon: IARC Press; 2008.

4. Advani R. Optimal therapy of advanced Hodgkin lymphoma. Hematology Am Soc Hematol Educ Program. 2011;2011:310-6.

5. Sureda A, Constans M, Iriondo A, et al. Prognostic factors affecting long-term outcome after stem cell transplantation in Hodgkin's lymphoma autografted after a first relapse. Ann Oncol. 2005;16:625-33.

6. Stein H, Foss HD, Dürkop H, et al. CD30(+) anaplastic large cell lymphoma: a review of its histopathologic, genetic, and clinical features. Blood. 2000;96:3681-95.

7. Kewalramani $\mathrm{T}$, Zelenetz AD, Teruya-Feldstein J, et al. Autologous transplantation for relapsed or primary refractory peripheral T-cell lymphoma. Br J Haematol. 2006;134:202-7.

8. Fanale MA, Younes A. Monoclonal antibodies in the treatment of non-Hodgkin's lymphoma. Drugs. 2007;67:333-50.

9. Bartlett NL, Younes A, Carabasi MH, et al. A Phase I multidose study of SGN-30 immunotherapy in patients with refractory or recurrent CD30+ hematologic malignancies. Blood. 2008;111: 1848-54.

10. Forero-Torres A, Leonard JP, Younes A, et al. A phase II study of SGN-30 (anti-CD30 mAb) in Hodgkin lymphoma or systemic anaplastic large cell lymphoma. Br J Haematol. 2009;146:171-9.

11. Duvic M, Reddy SA, Pinter-Brown L, et al. A phase II study of SGN-30 in cutaneous anaplastic large cell lymphoma and related lymphoproliferative disorders. Clin Cancer Res. 2009;15:6217-24.

12. Blum KA, Jung SH, Johnson JL, et al. Serious pulmonary toxicity in patients with Hodgkin's lymphoma treated with the anti-CD30 antibody, SGN-30, and gemcitabine, vinorelbine, and liposomal doxorubicin (GVD) is associated with a Fc $\gamma$ RIIIa-158 V/F polymorphism; results from CALGB 505502. Ann Oncol. 2010;21:2246-54.

13. Ansell SM, Horwitz SM, Engert A, et al. Phase I/II study of an anti-CD30 monoclonal antibody (MDX-060) in Hodgkin's lymphoma and anaplastic large cell lymphoma. J Clin Oncol. 2007;25:2764-9.

14. Thertulien R, Frankel AE, Evens AM, et al. A Phase I, open label, dose-escalation, multidose study of MDX-1401 (defucosylated human anti-CD30 monoclonal antibody) in patients with CD30positive refractory/relapsed Hodgkin's lymphoma. AACR Meeting Abstracts. 2009:Abstract 1227.

15. Blum KA, Smith M, Fung H, et al. Phase I study of an anti-CD30 Fc engineered humanized monoclonal antibody in Hodgkin lymphoma (HL) or anaplastic large cell lymphoma (ALCL) patients: safety, pharmacokinetics (PK), immunogenicity, and efficacy. J Clin Oncol. 2009;27(Suppl. 15):Abstract 8531. 
16. Francisco JA, Cerveny CG, Meyer DL, et al. cAC10vcMMAE, an anti-CD30 monomethyl auristatin E conjugate with potent and selective antitumor activity. Blood. 2003;102:1458-65.

17. Dubowchik GM, Firestone RA, Padilla L, et al. Cathepsin B-labile dipeptide linkers for lysosomal release of doxorubicin from internalizing immunoconjugates: model studies of enzymatic drug release and antigen-specific in vitro anticancer activity. Bioconjug Chem. 2002;13:855-69.

18. Oflazoglu E, Kissler JM, Sievers EL, et al. Combination of anti-CD30-auristatin-E antibody drug conjugate (SGN-35) with chemotherapy improves antitumor activity in Hodgkin lymphoma. Br J Haematol. 2008;142:69-73.

19. Younes A, Bartlett NL, Leonard JP, et al. Brentuximab vedotin (SGN-35) for relapsed CD-30 positive lymphomas. N Engl J Med. 2010;363: 1812-21.

20. Fanale MA, Forero-Torres A, Rosenblatt JD, et al. A phase I weekly dosing study of brentuximab vedotin in patients with relapsed/refractory CD30 positive hematologic malignancies. Clin Cancer Res. 2012;18:248-55.

21. Younes A, Gopal AK, Smith SE, et al. Results of a pivotal phase II study of brentuximab vedotin for patients with relapsed or refractory Hodgkin's lymphoma. J Clin Oncol. 2012;30:2197-203.

22. Pro B, Advani R, Brice $P$, et al. Brentuximab vedotin (SGN-35) in patients with relapsed or refractory systemic anaplastic large-cell lymphoma: results of a phase II study. J Clin Oncol. 2012;30:2190-6.

23. Ansell MS, Connors JM, Park SI, O'Meara M, Younes A. Frontline therapy with brentuximab vedotin combined with ABVD or AVD in patients with newly diagnosed advanced stage Hodgkin lymphoma. Blood (ASH Annual Meeting Abstracts). 2012;120:798.

24. Wagner-Johnston ND, Bartlett NL, Cashen A, Berger JR. Progressive multifocal leukoencephalopathy in a patient with Hodgkin lymphoma treated with brentuximab vedotin. Leuk Lymphoma. 2012;53:2283-6.

25. FDA Drug Safety Communication: New Boxed Warning and Contraindication for Adcetris (brentuximabvedotin). Food and Drug Administration website. http://www.fda.gov/ Drugs/DrugSafety/ucm287668.htm. Accessed 6 Feb 2013.

26. Gopal AK, Ramchandren R, O'Connor OA, et al. Safety and efficacy of brentuximab vedotin for
Hodgkin lymphoma recurring after allogeneic stem cell transplantation. Blood. 2012;120:560-8.

27. Seattle Genetics, Inc. Brentuximab vedotin in patients with CD30-positive nonlymphomatous malignancies. NCT01461538. ClinicalTrials.gov website. http://www.clinicaltrials.gov/ct2/show/ NCT01461538. Accessed 6 Feb 2013.

28. Seattle Genetics, Inc. A Phase 1 Study of Brentuximab Vedotin Given Sequentially and Combined With Multi-Agent Chemotherapy for CD30-Positive Mature T-Cell and NK-Cell Neoplasms. NCT01309789. ClinicalTrials.gov website. http://www.clinicaltrials.gov/ct2/show/ NCT01309789. Accessed 6 Feb 2013.

29. Fred Hutchinson Cancer Research Center/ University of Washington Cancer Consortium Brentuximab Vedotin in Treating Patients With Steroid-Resistant Acute Graft-Versus-Host Disease. NCT01616680. ClinicalTrials.gov website. http:// www.clinicaltrials.gov/ct2/show/NCT01616680. Accessed 6 Feb 2013.

30. Younes A, Sureda A, Ben-Yehuda D, et al. Panobinostat in patients with relapsed/refractory Hodgkin's lymphoma after autologous stem-cell transplantation: results of a phase II study. J Clin Oncol. 2012;30:2197-203.

31. Johnston PB, Inwards DJ, Colgan JP, et al. A phase II trial of the oral mTOR inhibitor everolimus in relapsed Hodgkin lymphoma. Am J Hematol. 2010; $85: 320-4$.

32. Fehniger TA, Larson S, Trinkaus $\mathrm{K}$, et al. A phase 2 multicenter study of lenalidomide in relapsed or refractory classical Hodgkin lymphoma. Blood. 2011;118:5119-25.

33. Younes A, Romaguera J, Hagemeister F, et al. A pilot study of rituximab in patients with recurrent classic Hodgkin disease. Cancer. 2003;98:310-4.

34. Coiffier B, Pro B, Prince HM, et al. Final results from a pivotal, multicenter, international, open-label, phase 2 study of romidepsin in progressive or relapsed peripheral T-cell lymphoma (PTCL) following prior systemic therapy. Blood. 2010; 116:114.

35. O'Connor OA, Pro B, Pinter-Brown L, et al. Pralatrexate in patients with relapsed or refractory peripheral T-cell lymphoma: results from the pivotal PROPEL study. J Clin Oncol. 2011;29:1182-9.

36. Bartlett N, Grove LE, Kennedy DA, Sievers EL, Forero-Torres A. Objective responses with brentuximab vedotin (SGN-35) retreatment in CD30-positive hematologic malignancies: A case series. J Clin Oncol. 2010(Suppl. 15):Abstract 8062. 